心脏病学

Am J Cardiol

\section{急性心肌梗死患者低密度脂蛋白胆 固醇越低，越不容易发生院内死亡}

晋川

众所周知，血脂代谢紊乱是急性心肌梗 死（AMI）的危险因子，特别是低密度脂蛋 白胆固醇 ( LDL-C ) 和高密度脂蛋白胆固醇 （ HDL-C ）。随着 LDL-C 水平的增高，患者 将来发生 AMI 的风险也随之增高。与 $\mathrm{LDL}-\mathrm{C}$ 不同的是, HDL-C 则是 AMI 的保护因子, 表现为 HDL-C 水平越高，患者越不容易发生 AMI。然而，在 AMI 发生与发展的过程中, LDL-C 和 HDL-C 除了作为危险 (保护) 因子外, 是否还可以作为预后因子呢?

近期 Am J Cardiol 在线刊登的一篇论文指 出, LDL-C 与 AMI 患者院内死亡密切相关。 研究者对美国国家心肌梗死登记中心 ( National Registry of Myocardial Infarction, NRMI ) 的数 据库进行了分析，采用多元 logistic 回归评价 了 115492 名 AMI 患者就诊 24 小时内的血脂水 平与院内死亡的关系。研究人员发现: LDL-C 水平越高，患者发生院内死亡的风险越小。 多变量的 logistic 回归结果表明：与 LDL-C 小 于 $77 \mathrm{mg} / \mathrm{dl}$ 的患者相比, LDL-C 大于 $128 \mathrm{mg} /$ $\mathrm{dl}$ 的患者发生院内死亡的优势比 ( OR ) 为 0.85 （95\%CI: 0.76-0.96）。最重要的是，LDL-C 与 AMI 患者院内死亡的关联性与患者发病前 是否接受降脂药物治疗无关，即不论患者人院 前是否接受过降脂药物治疗, LDL-C 增高均 与院内死亡风险降低有关。相比之下，HDL-C 与患者院内死亡的关系并不十分明显。

研究者指出, 在 AMI 发生与发展的过程中, 患者可能发生继发性肝脏功能衰竭, LDL-C 的 合成能力随之降低。因此, LDL-C 的水平在 一定程度上反映了肝脏的受损程度，其水平越
低，表明肝脏受损程度越严重，患者的预后也 因此越差。该研究发现 LDL-C 与 AMI 患者的 近期预后密切相关, 提示在 AMI 的救治过程中, 可以将 LDL-C 视为一个近期预后判断指标。

\section{原文链接}

http://www.sciencedirect.com/science/article/pii S0002914914022693

\section{D. anss \\ Blood \\ 血小板输注增加动脉栓塞和院内死 亡风险}

晋川

对于血小板减少的病人，比如免疫性血小 板减少性紫癜（ITP）、血栓性血小板减少性 紫癜( TTP )和肝素诱导的血小板减少症( HIT ), 血小板输注是较为常用但颇具争议的方法之 一，主要原因是对其安全性知之甚少。

众所周知，血小板是一期止血的中坚力量, 参与了血栓的形成。对于血小板输注，临床医 师最担心的是输注血小板是否会增加患者血栓 形成的风险。

近期 Blood 上刊登的一篇来自约翰霍普金 斯大学的研究对此进行了深人的解析。研究人 员利用全美住院病人数据库 (NIS) 对 20072011 年 的 79980 例 ITP、10624 名 TTP 和 6332 名 HIT 患者的住院病例进行了分析。

他们发现 $10.1 \%$ 的 TTP 患者、 $7.1 \%$ 的 HIT 患者和 $25.8 \%$ 的 ITP 患者曾接受过血小板 输注。在 TTP、HIT 和 ITP 患者中, 血栓的发 生率分别为 $4.1 \% 、 20.6 \%$ 和 $0.9 \%$; 出血的发 生率分别为 $13.7 \%$ 、 $5.7 \%$ 和 $11.5 \%$; 急性心肌 梗死的发生率分别为 $5.1 \% 、 7.1 \%$ 和 $0.5 \%$; 卒 中的发生率分别为 $5.2 \% 、 2.3 \%$ 和 $0.3 \%$ 。

多参数的 logistic 分析结果表明: 在 TTP 患者中，接受血小板输注会导致动脉栓塞、急 
性心肌梗死和院内死亡的风险显著增高, 但不 会增加卒中和静脉栓塞的风险; 在 HIT 患者中, 接受血小板输注会导致动脉栓塞的风险增高, 但不会增加静脉栓塞、急性心肌梗死和院内死 亡的风险; 在 ITP 患者中, 输注血小板并不会 增加静脉栓塞、动脉栓塞、急性心肌梗死、卒 中和院内死亡的风险。

该研究结果表明, 在 ITP 患者中, 输注血 小板是相对安全的, 不会增加血栓风险。但是 惕血栓的形成，在决定输注血小板时一定要权 衡利弊。

为何在 TTP 患者和 HIT 患者中输注血小 板会增加血栓风险，但是在 ITP 患者中输注 血小板却不会增加血栓风险呢? 研究人员解释 到: TTP 发生的主要病理生理特征为血小板微 血管血栓的形成; HIT 的发生则主要是由于肝 素依赖性的抗血小板因子导致血小板异常活化 和血栓形成。因此，在这类疾病中输注血小板 可能是一种 “火上浇油” 的行为, 需要十分谨 慎。

\section{原文链接}

http://www.bloodjournal.org/content/early/2015/01/21/ blood-2014-10-605493.long?sso-checked=true 在 HIT 和 TTP 患者中, 输注血小板时需要警

的基本结构是通过形成与消除而处于动态的变 化状态, 这是我们常说的可塑性。而在退行性 神经病变的病理情况下, 突触会减少, 从而导 致神经元间的联系受到损害。在冬眠动物中, 突触会在冬眠期间减少, 随着冬眠结束而重新 增加, 而这些变化伴随着大脑中一种蛋白质一 冷休克蛋白 RBM3 的变化。该现象促使英国 莱斯特大学的科学工作者试图寻找它们之间的 联系。

Mallucci 博士与他的团队在朊病毒 (prion ) 感染的小鼠 (海绵状脑组织病变模型) 和 5XFAD 小鼠（一种阿尔茨海默氏病模型）中 发现低温可导致突触的丢失和 RBM3 蛋白的 减少，提高 RBM3 蛋白的表达则可阻止低温 导致的突触丧失、防止小鼠出现行为缺陷和神 经元的丧失，并延长其生存期。相反，应用基 因敲除技术降低 RBM3 蛋白的表达则起到相 反的效果。

该研究将科学工作者对退行性神经病变的 认识推进了一大步, 可能促使人们寻找一种能 够改变 RBM3 蛋白表达的物质来保护神经元 的病变和突触的丢失。

原文链接

http://www.nature.com/nature/journal/vaop/ncurrent/full/ nature14142.html

\section{(1) 心脏病学 BMJ \\ 退行性神经病变研究的新方向 低温与冷休克蛋白 RBM3 \\ 罗氏 Elecsys 高敏肌钻蛋白 T 诊 断急性心肌梗死的 meta 分析}

Dr. Yufeng Xie

晋川

低温与冬眠的对神经的保护作用已经为人 熟知, 但是其保护作用除了能降低氧耗和能量 代谢外，我们对其发挥作用的分子机制并不是 十分清楚。最近发表在《自然》的一项研究揭 示了其机制可能与蛋白质 RBM3 有关。

在健康肌体中, 突触 - 神经元间相互联系
自肌钙蛋白被发现以来, 急性心肌梗死 ( AMI) 的诊断有了革命性的变化。值得注意 的是, 传统的肌钙蛋白检测法的检测下限过高, 在 AMI 发作早期, 肌钙蛋白水平往往低于检 